\title{
Distribuição das espécies e estrutura do manguezal do rio Benevente, Anchieta, ES
}

\author{
Detony José Calenzani Petri ${ }^{1}$, Elaine Bernini ${ }^{1,3}$, Leandro Marelli de Souza ${ }^{2}$ \& Carlos Eduardo Rezende ${ }^{1}$ \\ ${ }^{1}$ Laboratório de Ciências Ambientais, Centro de Biociências e Biotecnologia, \\ Universidade Estadual do Norte Fluminense - UENF, Av. Alberto Lamego, 2000, \\ Parque Califórnia, CEP 28013-602, Campos dos Goytacazes, RJ, Brasil \\ ${ }^{2}$ Escola Superior de Agricultura Luiz de Queiroz, Universidade de São Paulo - USP, \\ Campus Piracicaba, Av. Pádua Dias, 11, CEP 13418-900, Piracicaba, SP, Brasil \\ ${ }^{3}$ Autor para correspondência: Elaine Bernini,e-mail: elainebernini@ hotmail.com
}

PETRI, D.J.C., BERNINI, E., SOUZA, L.M. \& REZENDE, C.E. Species distribution and structure of mangrove of the Benevente River, Anchieta, ES. Biota Neotrop. 11(3): http://www.biotaneotropica.org.br/v11n3/en/abs tract?inventory+bn02011032011

\begin{abstract}
The structure of the mangrove forest of the Benevente River estuary, in Anchieta, ES, was analyzed by the plot method in order to evaluate the distribution of species along the river. The results indicated a trend of species distribution, in which Avicennia schaueriana Leechm ex Stapf \& Moldenke and Rhizophora mangle L. exhibited the highest dominance and relative density in areas under greater marine influence, while Laguncularia racemosa (L.) Gaertn.f. and Avicennia germinans (L.) Stearn. showed higher dominance and relative density in areas under greater influence of fresh water. The density ranged from 700 to 7,866 stems.ha $^{-1}$, the basal area from 10.3 to 72.2 $\mathrm{m}^{2}$.ha ${ }^{-1}$, the average height from 2.0 to $16.4 \mathrm{~m}$ and average DBH (diameter at breast height) from 4.7 to $27.3 \mathrm{~cm}$. Most forests exhibited distribution of trunks of the inverted iota type, with an absence of selective cutting. The contribution of the basal area was larger in diameter class $\geq 10 \mathrm{~cm}$, indicating the high structural development of the forests studied. The forests studied exhibited good conservation.
\end{abstract}

Keywords: phytosociology, manguezal, Avicennia, Laguncularia, Rhizophora.

PETRI, D.J.C., BERNINI, E., SOUZA, L.M. \& REZENDE, C.E. Distribuição das espécies e estrutura do manguezal do rio Benevente, Anchieta, ES. Biota Neotrop. 11(3): http://www.biotaneotropica.org.br/v11n3/ pt/abstract?inventory+bn02011032011

Resumo: A estrutura da floresta de mangue do estuário do rio Benevente, Anchieta, ES foi analisada pelo método de parcelas, com o propósito de avaliar a distribuição das espécies ao longo do rio. Os resultados indicaram uma tendência de distribuição das espécies, visto que Avicennia schaueriana Stapf \& Leechm ex Moldenke e Rhizophora mangle L. exibiram maior dominância e densidade relativa nas áreas sob maior influência marinha, enquanto que Laguncularia racemosa (L.) Gaertn.f. e Avicennia germinans (L.) Stearn. apresentaram maior densidade nas áreas sob maior influência de água doce. A densidade variou de 700 a 7.866 troncos.ha ${ }^{-1}$, a área basal de 10,3 a 72,2 $\mathrm{m}^{2} \cdot$ ha $^{-1}$, a altura média de 2,0 a 16,4 m e o DAP médio de 4,7 a 27,3 cm. A maioria das florestas analisadas exibiu distribuição de troncos do tipo jota invertido, com ausência de corte seletivo. A contribuição em área basal foi maior na classe diamétrica $\geq 10 \mathrm{~cm}$, indicando o elevado desenvolvimento estrutural das florestas estudadas. As florestas analisadas exibiram bom estado de conservação.

Palavras-chave: fitossociologia, manguezal, Avicennia, Laguncularia, Rhizophora. 


\section{Introdução}

A distribuição das espécies ao longo do gradiente de inundação tem sido amplamente abordada em estudos sobre manguezais e chamado a atenção dos cientistas há muito tempo (Watson 1928, Macnae 1968, Lugo \& Snedaker 1974, Snedaker 1982, Smith 1992, Ellison et al. 2000, Bernini \& Rezende 2004). Embora a maioria dos trabalhos descreva a distribuição das espécies de mangue ao longo do gradiente de inundação, alguns estudos também apresentam as tendências de distribuição ao longo do rio (Williams et al. 1991, Duke 1992, Bunt 1996, Bunt \& Stieglitz 1999, Silva et al. 2005, Soares et al. 2008, Bernini \& Rezende 2010). A variação na distribuição das espécies, tanto ao longo do gradiente de inundação quanto ao longo do rio, tem sido atribuída às respostas individuais das espécies às variações de inundação pelas marés, salinidade ou outras variáveis que se alteram previsivelmente ao longo do gradiente de inundação, além de fatores bióticos, como competição interespecífica e predação de diásporos (Ball 1980, Snedaker 1982, Smith 1987, 1992).

Os manguezais do Estado do Espírito Santo ocupam área aproximada de $70 \mathrm{~km}^{2}$ e se distribuem desde o riacho Doce, no extremo Norte, até o rio Itabapoana, na divisa com o Estado do Rio de Janeiro (Vale \& Ferreira 1998). Neste Estado, a estrutura da vegetação foi analisada nos manguezais do rio São Mateus (Vale 1999, Silva et al. 2005), Baía de Vitória (Carmo et al. 1995, 1998a), rio Reis Magos (Carmo et al. 1998b), rio Benevente (Pereira et al. 2009) e rio Itabapoana (Bernini \& Rezende 2010). No estuário do rio São Mateus (Vale 1999, Silva et al. 2005) e no estuário do rio Itabapoana (Bernini \& Rezende 2010) foram observadas tendências na distribuição das espécies de mangue, com algumas exibindo maior abundância nas áreas mais próximas do Oceano e outras em locais com menor influência marinha.

O manguezal que se desenvolve no estuário do rio Benevente é um dos mais extensos e conservados do Espírito Santo, com área aproximada de 4,6 $\mathrm{km}^{2}$ (Vale \& Ferreira 1998). Neste estuário, Pereira et al. (2009) descreveram a estrutura do manguezal citando Rhizophora mangle L. como espécie dominante e mais abundante em sítios situados na embocadura do rio. No entanto, os autores não estudaram tendências de distribuição das espécies ao longo do rio. As espécies podem exibir tendências na distribuição ao longo dos rios, em virtude da variação de fatores bióticos e abióticos (Ball 1980, Santos 1986, Mckee 1993, Silva et al. 2005, Soares et al. 2008, Bernini \& Rezende 2010). Considerando que o manguezal do rio Benevente estende-se por cerca de $6,5 \mathrm{~km}$ continente adentro, espera-se encontrar tendências de distribuição das espécies em virtude da variação das condições ambientais. Assim, o objetivo deste estudo é caracterizar a estrutura da floresta de mangue do estuário do rio Benevente, com o propósito de avaliar a distribuição das espécies ao longo do rio.

\section{Material e Métodos}

A bacia do rio Benevente drena área equivalente a $1.190 \mathrm{~km}^{2}$, incluindo os municípios de Alfredo Chaves, Anchieta e parte dos municípios de Piúma, Iconha e Guarapari, sendo também uma importante fonte de abastecimento de água para as cidades que integram a grande Vitória. Este rio deságua em Anchieta, que está localizada no litoral Sul do Estado Espírito Santo a $80 \mathrm{~km}$ da capital Vitória (Figura 1). Nas áreas do entorno do manguezal que se desenvolve no estuário do rio Benevente, com destaque para a parte superior do estuário com maior influência de água doce, vê-se claramente a mudança da paisagem na transição para outros ecossistemas, resquícios de restinga e mata atlântica. Contudo, parte da área de restinga e mata atlântica é ocupada por pastagens (criação de gado) e há algumas áreas onde se pratica a silvicultura (eucalipto) para produção de madeira. Na parte inferior do estuário ocorre pouca ocupação urbana na área de manguezal com apenas algumas moradias próximas das áreas de mangue e restinga.

O estudo foi conduzido no período de agosto de 2005 a novembro de 2008. A caracterização da estrutura da vegetação foi realizada através do método de parcelas, de acordo com a metodologia descrita em Schaeffer-Novelli \& Cintrón (1986). Para a amostragem da vegetação foram selecionados 20 sítios de estudo, variando de 400 a $800 \mathrm{~m}$ entre si. A demarcação ocorreu até $6,5 \mathrm{~km}$ de extensão, limite do manguezal continente adentro, considerando-se a representatividade geral do manguezal e a facilidade de acesso às áreas (Tabela 1 e Figura 1). Em cada sítio demarcou-se uma parcela ( $5 \mathrm{~m}$ da margem do canal) cuja área variou de 100 a $650 \mathrm{~m}^{2}$, de acordo com a densidade da vegetação (incluindo no mínimo 30 indivíduos vivos), correspondendo a uma área total de 0,635 ha. Dentro de cada parcela, todas as árvores maiores que 1,0 m de altura tiveram altura (telêmetro óptico) e a circunferência (a 1,30 m do substrato; fita métrica) medidas. No caso dos indivíduos menores que $1,30 \mathrm{~m}$ de altura, foram medidos o diâmetro do tronco abaixo da primeira ramificação (Soares 1999). A circunferência de árvores mortas e ainda em pé também foi incluída na amostragem.

Posteriormente, os dados de circunferência dos troncos vivos e mortos foram transformados em diâmetro à altura do peito $(\mathrm{DAP}=\mathrm{CAP} / \pi)$. Os parâmetros estruturais calculados foram a altura média, o DAP médio, a área basal de indivíduos vivos e mortos por espécie e classe diamétrica, a densidade de troncos vivos e mortos, a dominância em área basal e a densidade relativa de troncos (SchaefferNovelli \& Cintrón 1986). A distribuição de troncos por classes de diâmetro foi feita por sítio de estudo. Através do programa Statistica 7.0, realizou-se uma análise de agrupamento (UPGMA) considerando a densidade relativa das espécies.

Tabela 1. Coordenadas geográficas dos sítios selecionados no manguezal do estuário do rio Benevente, Espírito Santo.

Table 1. Geographic coordinates of selected sites in the mangrove forest of the estuary of Benevente River, Espírito Santo.

\begin{tabular}{|c|c|c|c|}
\hline Sítio & Coordenada geográfica & Sítio & Coordenada geográfica \\
\hline $\mathrm{S} 1$ & $20^{\circ} 48^{\prime} 68^{\prime \prime} \mathrm{S}$ e $40^{\circ} 39^{\prime} 83^{\prime \prime} \mathrm{O}$ & S11 & $20^{\circ} 46^{\prime} 47^{\prime \prime} \mathrm{S}$ e $40^{\circ} 38^{\prime} 80^{\prime \prime} \mathrm{O}$ \\
\hline $\mathrm{S} 2$ & $20^{\circ} 48^{\prime} 41^{\prime \prime} \mathrm{S}$ e $40^{\circ} 39^{\prime} 83^{\prime \prime} \mathrm{O}$ & $\mathrm{S} 12$ & $20^{\circ} 46^{\prime} 34^{\prime \prime} \mathrm{S}$ e $40^{\circ} 38^{\prime} 98^{\prime \prime} \mathrm{O}$ \\
\hline S3 & $20^{\circ} 48^{\prime} 08^{\prime \prime} \mathrm{S}$ e $40^{\circ} 39^{\prime} 94^{\prime \prime} \mathrm{O}$ & $\mathrm{S} 13$ & $20^{\circ} 46^{\prime} 43^{\prime \prime} \mathrm{S}$ e $40^{\circ} 46^{\prime} 53^{\prime \prime} \mathrm{O}$ \\
\hline $\mathrm{S} 4$ & $20^{\circ} 47^{\prime} 79^{\prime \prime} \mathrm{S}$ e $40^{\circ} 39^{\prime} 67^{\prime \prime} \mathrm{O}$ & S14 & $20^{\circ} 46^{\prime} 74^{\prime \prime} \mathrm{S}$ e $40^{\circ} 38^{\prime} 01^{\prime \prime} \mathrm{O}$ \\
\hline S5 & $20^{\circ} 47^{\prime} 41^{\prime \prime} \mathrm{S}$ e $40^{\circ} 39^{\prime} 53 " \mathrm{O}$ & $\mathrm{S} 15$ & $20^{\circ} 45^{\prime} 83^{\prime \prime} \mathrm{S}$ e $40^{\circ} 38^{\prime} 20^{\prime \prime} \mathrm{O}$ \\
\hline S6 & $20^{\circ} 47^{\prime} 68^{\prime \prime} \mathrm{S}$ e $40^{\circ} 38^{\prime} 11^{\prime \prime} \mathrm{O}$ & S16 & $20^{\circ} 46^{\prime} 08^{\prime \prime} \mathrm{S}$ e $40^{\circ} 38^{\prime} 78^{\prime \prime} \mathrm{O}$ \\
\hline S7 & $20^{\circ} 47^{\prime} 22^{\prime \prime} \mathrm{S}$ e $40^{\circ} 38^{\prime} 37^{\prime \prime} \mathrm{O}$ & S17 & $20^{\circ} 46^{\prime} 18^{\prime \prime} \mathrm{S}$ e $40^{\circ} 38^{\prime} 80^{\prime \prime} \mathrm{O}$ \\
\hline S8 & $20^{\circ} 47^{\prime} 32^{\prime \prime} \mathrm{S}$ e $40^{\circ} 39^{\prime} 58^{\prime \prime} \mathrm{O}$ & $\mathrm{S} 18$ & $20^{\circ} 46^{\prime} 69^{\prime \prime} \mathrm{S}$ e $40^{\circ} 39^{\prime} 27^{\prime \prime} \mathrm{O}$ \\
\hline S9 & $20^{\circ} 47^{\prime} 34^{\prime \prime} \mathrm{S}$ e $40^{\circ} 39^{\prime} 83^{\prime \prime} \mathrm{O}$ & S19 & $20^{\circ} 46^{\prime} 69^{\prime \prime} \mathrm{S}$ e $40^{\circ} 39^{\prime} 43^{\prime \prime} \mathrm{O}$ \\
\hline S10 & $20^{\circ} 47^{\prime} 09 " \mathrm{~S}$ e $40^{\circ} 38^{\prime} 97^{\prime \prime} \mathrm{O}$ & S20 & $20^{\circ} 46^{\prime} 76^{\prime \prime} \mathrm{S}$ e $40^{\circ} 39^{\prime} 99^{\prime \prime} \mathrm{O}$ \\
\hline
\end{tabular}



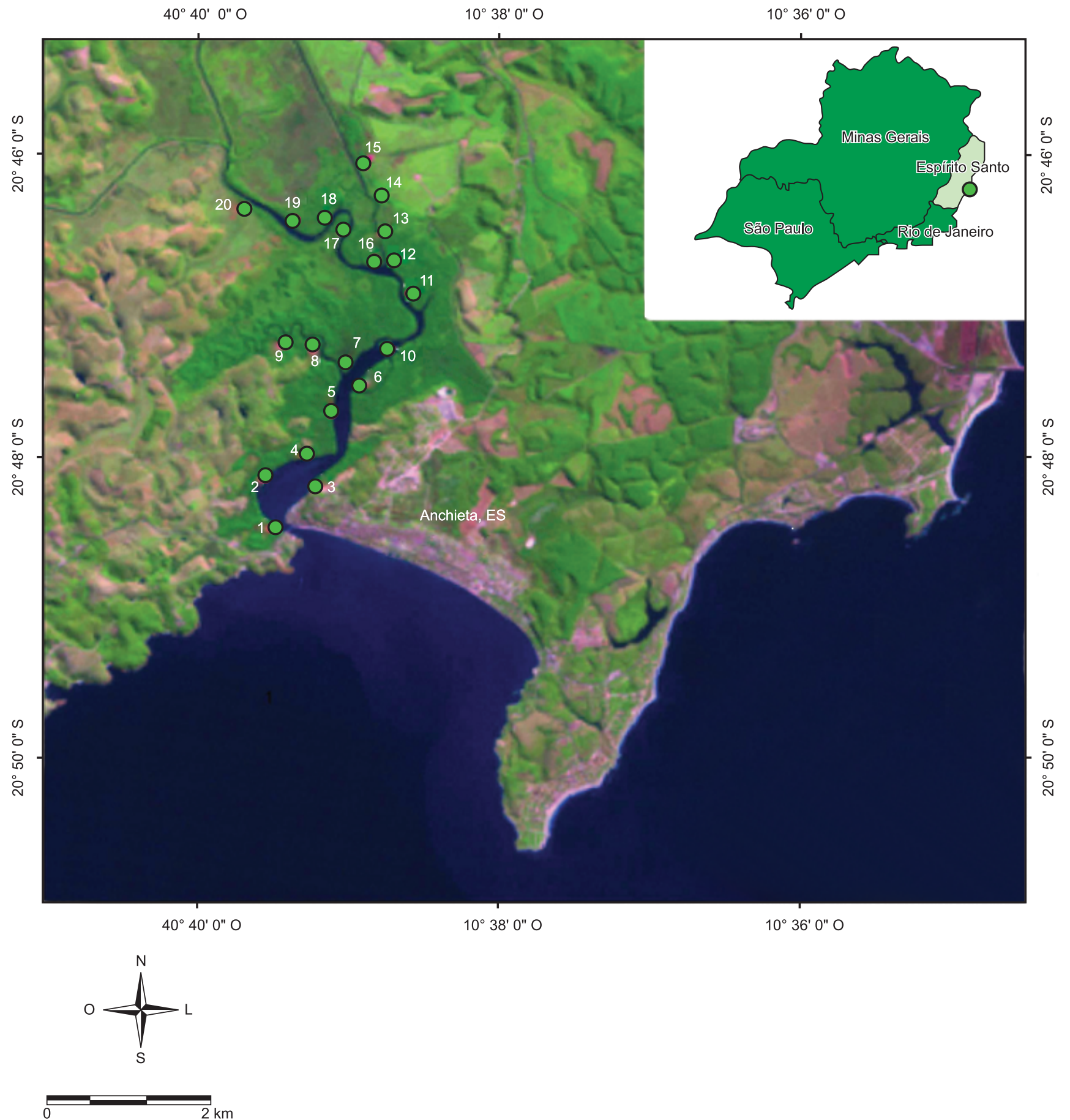

Figura 1. Sítios de estudo analisados no manguezal do estuário do rio Benevente, Anchieta, Espírito Santo.

Figure 1. Study sites analyzed in the mangrove of the estuary of the Benevente, Espírito Santo.

\section{Resultados}

A densidade de troncos vivos variou de 700 a 7.866 troncos $^{-1} a^{-1}$ e a de troncos mortos de 20 a 3.700 troncos.ha-1 (Tabela 2). Baseado nos dados de densidade relativa, a análise de agrupamento identificou dois grandes grupos (Figura 2). O primeiro grupo, composto pelos sítios S20, S19, S18, S13, S15, S17, S14, S12, S16 e S8, apresentou maior densidade relativa de A. germinans ou L. racemosa (Figura 3), com exceção dos sítios S8 e S16 que não apresentaram valores elevados de densidade relativa para uma única espécie. O segundo grande grupo é formado pelos sítios $\mathrm{S} 10, \mathrm{~S} 5, \mathrm{~S} 4, \mathrm{~S} 9, \mathrm{~S} 2, \mathrm{~S} 11, \mathrm{~S} 3$, $\mathrm{S} 7, \mathrm{~S} 6$ e S1 e exibiram maior densidade relativa de A. schaueriana ou $R$. mangle (Figura 3). Neste grupo, identificou-se um subgrupo com maior densidade relativa de $A$. schaueriana (S10, S5, S4, S9 e $\mathrm{S} 2)$ e outro subgrupo com maior densidade relativa de $R$. mangle (S11, S3, S7, S6 e S1).

A distribuição dos troncos vivos e mortos em classes de diâmetro (Figuras 4 e 5) indicou que 12 sítios (S2, S4, S9, S10, S12, S13, S14, S15, S16, S17, S18 e S19) exibiram distribuição no formato de jota invertido. Os demais sítios (S1, S3, S5, S6, S7, S8, S11 e S20) 
Petri, D.J.C. et al.

Tabela 2. Parâmetros estruturais da floresta de mangue do estuário do rio Benevente, Espírito Santo.

Table 2. Structural parameters in the mangrove forest of the estuary of Benevente River, Espírito Santo.

\begin{tabular}{|c|c|c|c|c|c|c|}
\hline Sítio & DTv ${ }^{1}$ & DTm $^{1}$ & $\mathbf{A B v ^ { 2 }}$ & $\mathbf{A B m}^{2}$ & Altura média ${ }^{3}$ & DAP médio \\
\hline S1 & 1.178 & 156 & 22,8 & 1,18 & $11,2 \pm 5,6$ & 15,7 \\
\hline S2 & 2.760 & 160 & 26,4 & 0,53 & $8,5 \pm 7,6$ & 11,0 \\
\hline S3 & 7.866 & 167 & 13,4 & 2,17 & $2,0 \pm 3,2$ & 4,7 \\
\hline S4 & 1.700 & 133 & 36,1 & 0,54 & $11,0 \pm 8,8$ & 16,4 \\
\hline S5 & 1.000 & 200 & 23,6 & 2,23 & $11,0 \pm 7,8$ & 17,3 \\
\hline S6 & 720 & 60 & 18,8 & 0,66 & $15,7 \pm 9,3$ & 18,2 \\
\hline S7 & 700 & 20 & 41,1 & 0,01 & $16,9 \pm 8,1$ & 27,3 \\
\hline S8 & 1.433 & 600 & 25,1 & 11,06 & $11,0 \pm 7,9$ & 14,9 \\
\hline S9 & 1.500 & 480 & 10,3 & 0,72 & $6,8 \pm 4,1$ & 9,3 \\
\hline S10 & 6.300 & 1.600 & 17,4 & 0,67 & $9,1 \pm 4,7$ & 5,9 \\
\hline S11 & 1.300 & 33 & 21,3 & 2,74 & $16,4 \pm 5,8$ & 14,5 \\
\hline S12 & 5.257 & 514 & 20,4 & 1,44 & $7,2 \pm 4,5$ & 7,0 \\
\hline S13 & 6.900 & 1.300 & 72,2 & 3,09 & $5,4 \pm 5,2$ & 13,5 \\
\hline S14 & 6.550 & 2.200 & 23,2 & 2,87 & $8,6 \pm 4,6$ & 6,7 \\
\hline S15 & 6.800 & 3.700 & 18,8 & 0,54 & $7,1 \pm 2,1$ & 5,9 \\
\hline S16 & 1.875 & 225 & 45,7 & 1,23 & $9,4 \pm 6,3$ & 17,6 \\
\hline S17 & 2.867 & 533 & 25,0 & 2,10 & $6,6 \pm 5,4$ & 10,5 \\
\hline S18 & 6.800 & 933 & 46,6 & 5,21 & $7,7 \pm 2,9$ & 9,3 \\
\hline S19 & 1.200 & 100 & 16,2 & 0,01 & $9,4 \pm 6,3$ & 17,6 \\
\hline S20 & 740 & 140 & 21,4 & 2,28 & $13,8 \pm 7,0$ & 19,2 \\
\hline
\end{tabular}

DTv: densidade de troncos vivos; DTm: densidade de troncos mortos; ABv: área basal viva; ABm: área basal morta; DAP: diâmetro à altura do peito; Unidades para subscritos: $1 \mathrm{em}$ troncos ha $\mathrm{a}^{-1} ; 2 \mathrm{em} \mathrm{m}^{2}$ ha $^{-1} ; 3 \mathrm{em} \mathrm{m}$, média \pm desvio padrão; $4 \mathrm{em} \mathrm{cm}$.

DTV: density of living trunks; DTM density of dead trunks; ABV: basal area of living; ABm: basal area of dead; DBH: diameter at breast height; Units subscribed for: 1 in trunk ha ${ }^{-1} ; 2$ in $\mathrm{m}^{2} \mathrm{ha}^{-1}$, mean \pm standard deviation; 4 in $\mathrm{cm}$.

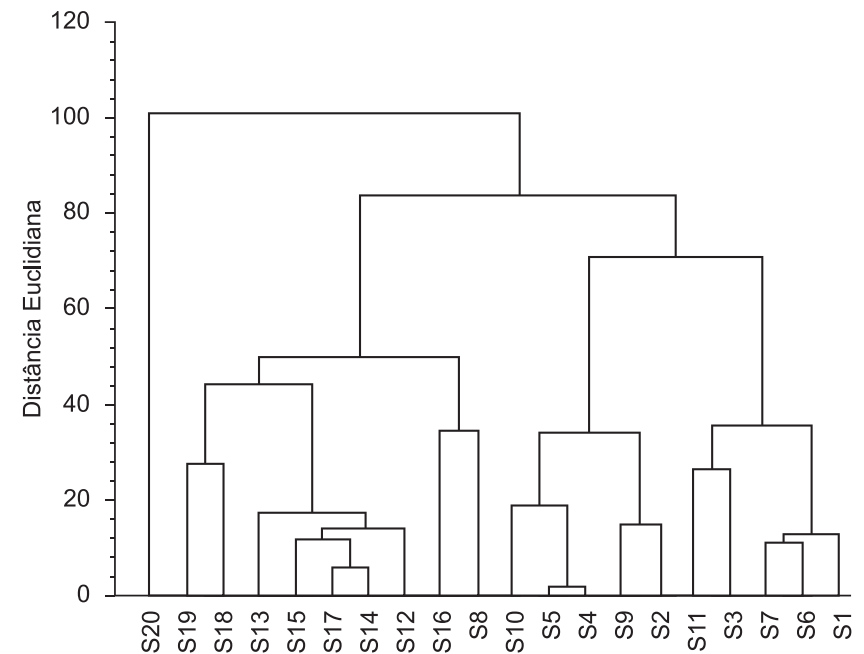

Figura 2. Análise de agrupamento (UPGMA) realizada para os dados de densidade relativa das espécies analisadas nos sítios $\mathrm{S} 1$ a S20, no manguezal do estuário do rio Benevente, Espírito Santo.

Figure 2. Cluster analysis (UPGMA) performed for the density data on the species analyzed in the sites S1 to S20, in the mangrove forest estuary of Benevente River, Espírito Santo.

apresentaram variação nas diferentes classes de diâmetro, com maior contribuição de troncos mortos nas classes de diâmetro inferiores ou intermediárias.

A área basal viva variou de 10,3 a $72,2 \mathrm{~m}^{2} \cdot \mathrm{ha}^{-1} \mathrm{e}$ a área basal morta variou de 0,01 a $11,06 \mathrm{~m}^{2} \cdot \mathrm{ha}^{-1}$ (Tabela 2). Com exceção do

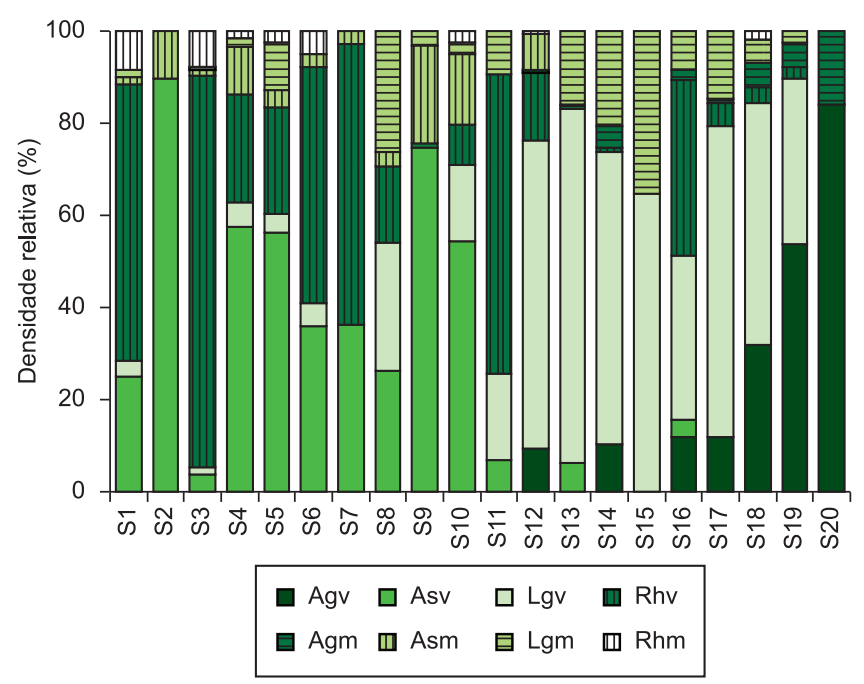

Figura 3. Densidade relativa de troncos vivos e mortos por espécie, no manguezal do estuário do rio Benevente, Espírito Santo. Agv: Avicennia germinans viva; Agm: Avicennia germinans morta; Asv: Avicennia schaueriana viva; Asm: Avicennia schaueriana morta; Lgv: Laguncularia racemosa viva; Lgm: Laguncularia racemosa morta; Rhv: Rhizophora mangle viva; Rhm: Rhizophora mangle morta.

Figure 3. Relative density of living and dead trunks by species in the mangrove forest estuary of Benevente River, Espírito Santo. Agv: Avicennia germinans alive; Agm: Avicennia germinans dead; Asv: Avicennia schaueriana alive; Asm: Avicennia schaueriana dead; Lgv: Laguncularia racemosa alive; Lgm: Laguncularia racemosa dead; Rhv: Rhizophora mangle alive; Rhm: Rhizophora mangle dead. 

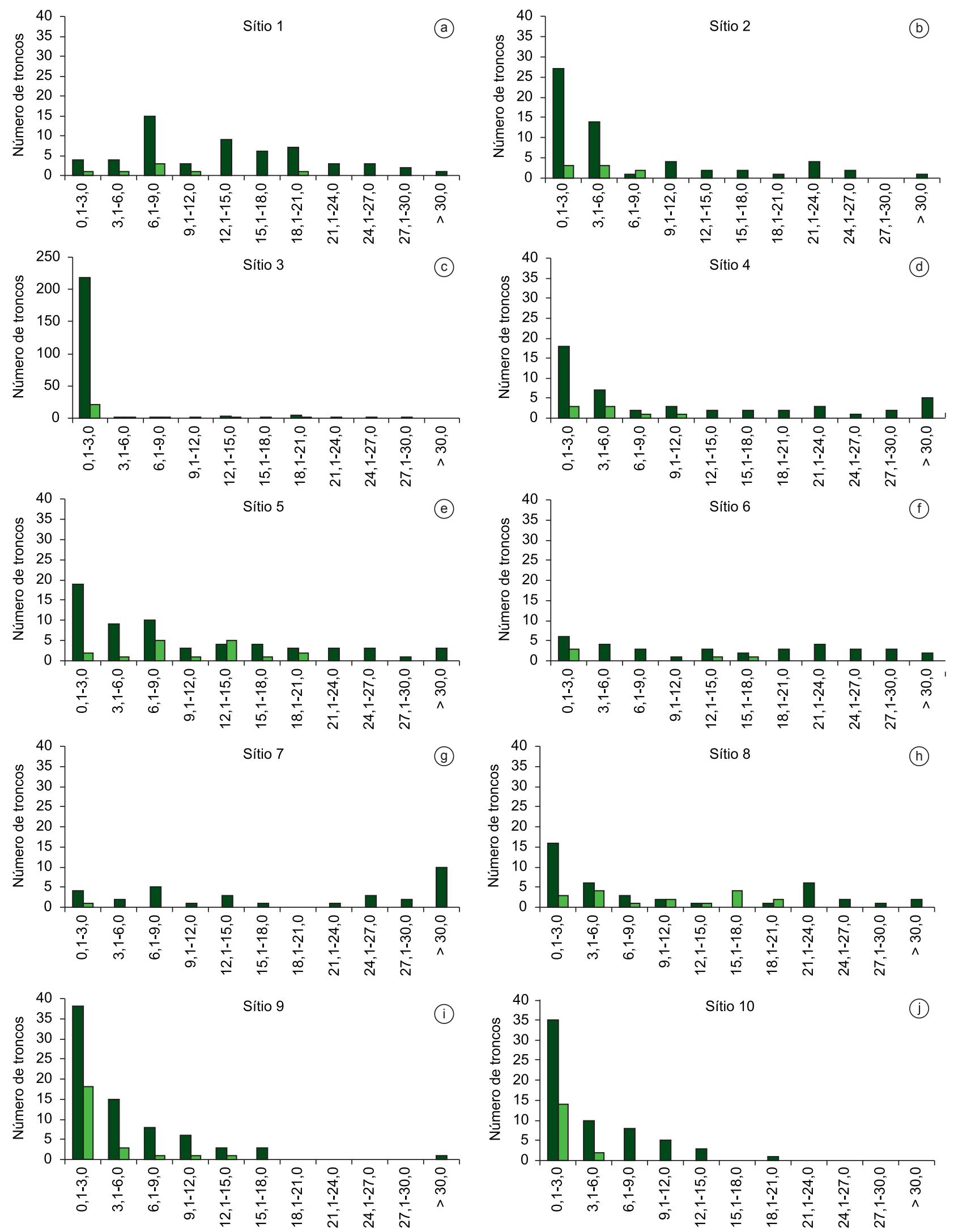

Figura 4. Distribuição de troncos vivos (verde escuro) e mortos (verde claro) por classes de diâmetro, nos sítios 1 a 10 analisados no manguezal do estuário do rio Benevente, Espírito Santo.

Figure 4. Distribution of live trunks (dark green) and dead trunks (light green) by diameter classes in sites from 1 to 10 analyzed in the mangrove of the estuary of the Benevente, Espírito Santo. 
Petri, D.J.C. et al.
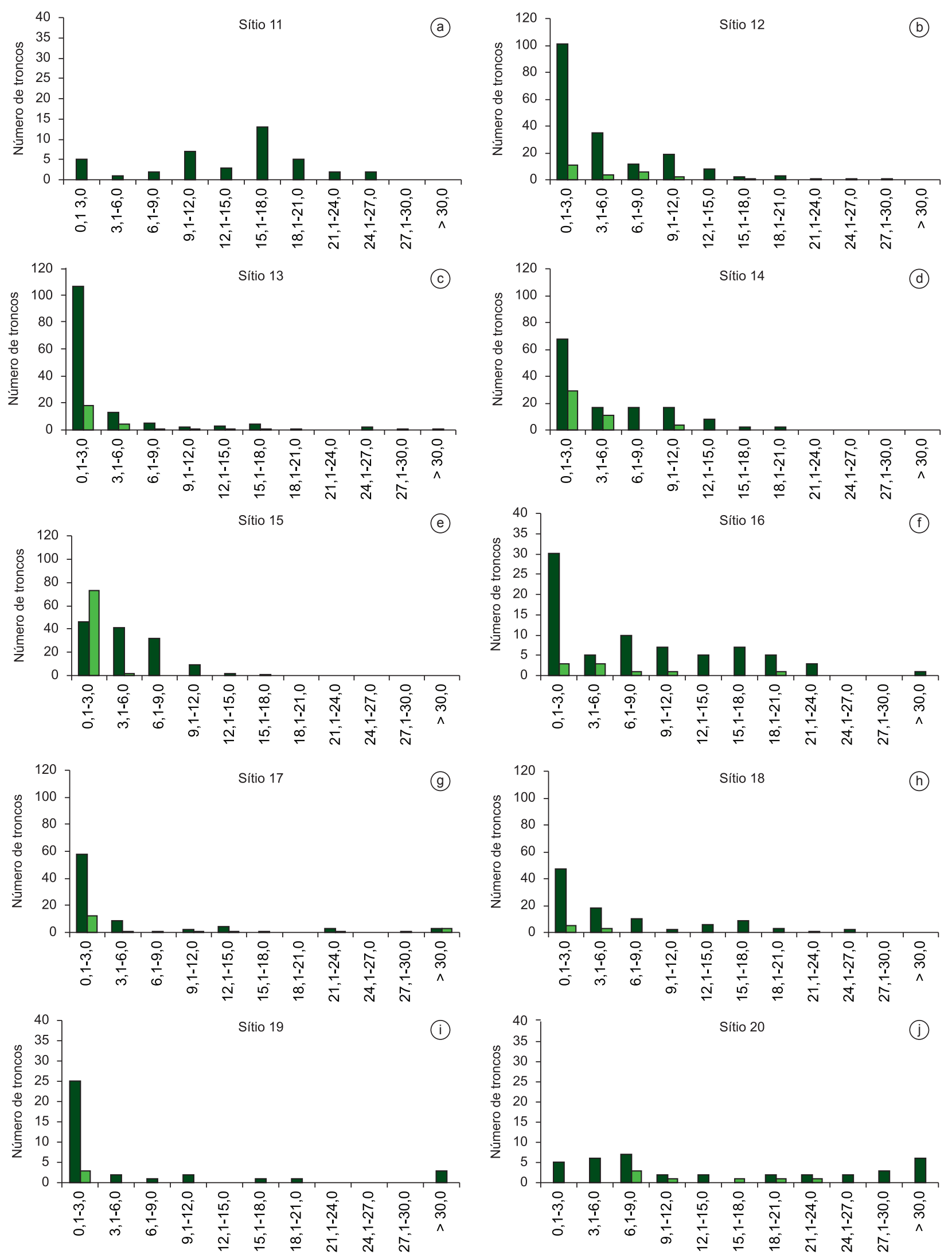

Figura 5. Distribuição de troncos vivos (verde escuro) e mortos (verde claro) por classes de diâmetro, nos sítios 11 a 20 analisados no manguezal do estuário do rio Benevente, Espírito Santo.

Figure 5. Distribution of live trunks (dark green) and dead trunks (light green) by diameter classes in sites from 1 to 10 analyzed in the mangrove of the estuary of the Benevente, Espírito Santo. 
S15, houve maior contribuição de dominância em área basal na classe diamétrica $\geq 10 \mathrm{~cm}$ (Tabela 3 ). A espécie com maior dominância em área basal viva foi A. schaueriana (44\%), seguida da $L$. racemosa (28\%), R. mangle (15\%) e A. germinans (13\%). Especificamente, A. schaueriana foi dominante nos sítios $\mathrm{S} 2, \mathrm{~S} 4, \mathrm{~S} 5, \mathrm{~S} 7, \mathrm{~S} 8, \mathrm{~S} 9, \mathrm{~S} 13$ e $\mathrm{S} 16$, L. racemosa nos sítios $\mathrm{S} 10, \mathrm{~S} 12, \mathrm{~S} 14, \mathrm{~S} 15$ e S18, $R$. mangle nos sítios S1, S3, S6 e S11 e A. germinans nos sítios S17, S19 e S20 (Tabela 4). A sequência de dominância em área basal morta (Tabela 4) foi $L$. racemosa (64\%), A. schaueriana (17\%), A. germinans (10\%) e $R$. mangle (9\%).

As alturas médias variaram de 2,0 a 16,9 m (Tabela 2). As maiores alturas individuais por espécie foram $27,0 \mathrm{~m}$ para A. schaueriana, $25,5 \mathrm{~m}$ para $R$. mangle, 23,0 m para A. germinans e $22,5 \mathrm{~m}$ para L. racemosa. Com relação ao DAP médio, os valores variaram de 4,7 a 27,3 cm (Tabela 2), sendo que os maiores valores individuais foram de 126, 41, 33 e $27 \mathrm{~cm}$ para A. schaueriana, A. germinans, $R$. mangle e $L$. racemosa, respectivamente.

Tabela 3. Área basal $\left(\mathrm{m}^{2} \cdot \mathrm{ha}^{-1}\right)$ viva e morta, por classe diamétrica $(\mathrm{cm})$, no manguezal do estuário do rio Benevente, Espírito Santo.

Table 3. Basal area $\left(\mathrm{m}^{2} . \mathrm{ha}^{-1}\right)$ alive and dead, by diameter class $(\mathrm{cm})$ in the mangrove forest of estuary of Benevente River, Espírito Santo.

\begin{tabular}{|c|c|c|c|c|c|c|}
\hline \multirow[t]{2}{*}{ Sítio } & \multicolumn{3}{|c|}{ Área basal viva } & \multicolumn{3}{|c|}{ Área basal morta } \\
\hline & $<2,5 \mathrm{~cm}$ & $\geq 2,5 \mathrm{~cm}$ & $\geq 10,0 \mathrm{~cm}$ & $<2,5 \mathrm{~cm}$ & $\geq 2,5 \mathrm{~cm}$ & $\geq 10,0 \mathrm{~cm}$ \\
\hline S1 & 0,02 & 1,46 & 21,34 & - & 0,52 & 0,66 \\
\hline S2 & 0,21 & 3,17 & 23,01 & 0,02 & 0,51 & - \\
\hline S3 & 0,56 & 0,41 & 12,39 & 0,03 & 0,19 & 1,95 \\
\hline S4 & 0,06 & 1,13 & 34,93 & 0,02 & 0,22 & 0,30 \\
\hline S5 & 0,04 & 0,96 & 22,58 & 0,01 & 0,29 & 1,94 \\
\hline S6 & 0,02 & 0,48 & 18,26 & 0,01 & - & 0,65 \\
\hline S7 & 0,01 & 0,57 & 40,49 & 0,01 & - & - \\
\hline S8 & 0,09 & 0,86 & 24,19 & 0,03 & 0,40 & 10,63 \\
\hline S9 & 0,13 & 2,01 & 8,11 & 0,05 & 0,25 & 0,42 \\
\hline $\mathrm{S} 10$ & 0,60 & 5,04 & 11,74 & 0,20 & 0,47 & - \\
\hline S11 & 0,01 & 0,86 & 20,47 & - & 0,17 & 2,57 \\
\hline $\mathrm{S} 12$ & 0,63 & 3,92 & 15,83 & 0,06 & 0,55 & 0,83 \\
\hline $\mathrm{S} 13$ & 0,83 & 2,35 & 69,00 & 0,19 & 0,70 & 2,20 \\
\hline $\mathrm{S} 14$ & 0,35 & 6,62 & 16,20 & 0,13 & 0,82 & 1,92 \\
\hline S15 & 0,27 & 14,89 & 3,60 & 0,42 & 0,12 & - \\
\hline S16 & 0,25 & 1,20 & 44,21 & 0,02 & 0,17 & 1,04 \\
\hline S17 & 0,28 & 0,85 & 23,92 & 0,03 & 0,10 & 1,97 \\
\hline $\mathrm{S} 18$ & 2,47 & 5,46 & 38,67 & 0,06 & 0,28 & 4,87 \\
\hline $\mathrm{S} 19$ & 0,12 & 0,31 & 15,77 & 0,01 & - & - \\
\hline $\mathrm{S} 20$ & 0,01 & 0,80 & 20,57 & - & 0,29 & 1,99 \\
\hline
\end{tabular}

Tabela 4. Dominância de troncos vivos e mortos (\%) das espécies analisadas no manguezal do estuário do rio Benevente, Espírito Santo.

Table 4. Dominance of living and dead trunks by species in the mangrove forest estuary of Benevente River, Espírito Santo.

\begin{tabular}{|c|c|c|c|c|c|c|c|c|}
\hline \multirow[t]{2}{*}{ Sítio } & \multicolumn{4}{|c|}{ Troncos vivos } & \multicolumn{4}{|c|}{ Troncos mortos } \\
\hline & Ag & As & Lg & $\mathbf{R h}$ & Ag & As & Lg & $\mathbf{R h}$ \\
\hline S1 & - & 27,71 & 0,03 & 67,34 & - & 0,32 & 0,62 & 3,98 \\
\hline S2 & - & 99,70 & - & - & - & 0,30 & - & - \\
\hline S3 & - & 31,11 & 1,54 & 53,36 & - & 3,23 & 0,51 & 10,25 \\
\hline S4 & - & 75,70 & 0,55 & 22,29 & - & 0,56 & 0,09 & 0,81 \\
\hline S5 & - & 65,43 & 0,78 & 25,15 & - & 0,09 & 7,56 & 0,99 \\
\hline S6 & - & 32,09 & 0,04 & 64,51 & - & 1,38 & - & 1,98 \\
\hline S7 & - & 52,47 & - & 47,51 & - & 0,02 & - & - \\
\hline S8 & - & 43,18 & 15,43 & 10,84 & - & 12,96 & 17,59 & - \\
\hline S9 & - & 92,69 & - & 0,73 & - & 2,01 & 4,57 & - \\
\hline S10 & - & 39,92 & 53,13 & 3,26 & - & 1,99 & 1,24 & 0,46 \\
\hline S11 & - & 7,52 & 16,33 & 64,78 & - & - & 11,37 & - \\
\hline S12 & 1,80 & - & 82,77 & 8,84 & 0,05 & - & 6,08 & 0,46 \\
\hline S13 & - & 80,77 & 14,15 & 0,98 & - & 0,80 & 3,30 & - \\
\hline S14 & 8,52 & - & 80,41 & 0,04 & 0,13 & - & 10,90 & - \\
\hline S15 & - & - & 97,22 & - & - & - & 2,78 & - \\
\hline S16 & 2,33 & 70,90 & 7,50 & 16,65 & 0,10 & - & 2,52 & - \\
\hline S17 & 67,83 & - & 23,87 & 0,57 & 1,13 & - & 6,60 & - \\
\hline S18 & 22,75 & - & 66,53 & 0,67 & 2,63 & - & 7,32 & 0,10 \\
\hline S19 & 86,28 & - & 11,03 & 2,62 & 0,02 & - & 0,05 & - \\
\hline $\mathrm{S} 20$ & 90,38 & - & - & - & 9,62 & - & - & - \\
\hline
\end{tabular}

Ag: Avicennia germinans; As: Avicennia schaueriana; Lg: Laguncularia racemosa; Rh: Rhizophora mangle.

Ag: Avicennia germinans; As: Avicennia schaueriana; Lg: Laguncularia racemosa; Rh: Rhizophora mangle. 


\section{Discussão}

No presente estudo, A. schaueriana e $R$. mangle exibiram maior densidade relativa na região mais próxima ao Oceano, enquanto que $L$. racemosa e A. germinans apresentaram maior densidade na região sob menor influência marinha. Silva et al. (2005) descreveram tendência similar no estuário do rio São Mateus (ES), enquanto que Soares et al. (2008) também citam A. schaueriana nas áreas com maior influência marinha e $L$ racemosa nas áreas com maior influência do aporte de água doce. Similarmente, o estudo da distribuição das espécies no litoral do Estado do Maranhão revelou tendências na distribuição de jusante para montante no estuário do rio Preguiças (MA), com a sequência: $R$. mangle, Rhizophora harrisonii Leechm.e L. racemosa (Santos 1986).

A. schaueriana é mais tolerante à salinidade (Cintrón et al. 1978, Tomlinson 1986) e apresenta melhor eficiência na utilização da água e do nitrogênio em relação à L. racemosa (Lovelock \& Feller 2003), o que explica sua dominância e densidade relativa elevada nas áreas mais próximas ao oceano. O gênero Avicennia pode ser encontrado dominando substratos altamente salinos ou em áreas sujeitas a baixas temperaturas, no entanto, é capaz de atingir melhor desenvolvimento estrutural em baixas salinidades (Cintrón-Molero \& Schaeffer-Novelli 1992). Este parece ser o caso da A. germinans no presente estudo, visto que esta espécie foi dominante na área sob menor influência salina.

Em campo foi possível observar que os substratos dos sítios $\mathrm{S} 1$, S3, S6 e S11 permanecem mais tempo inundados em relação aos demais, e a dominância e maior densidade relativa de $R$. mangle nestes sítios pode ser em parte a sua maior tolerância às condições de inundação (Mckee, 1993).

Alguns estudos, no entanto, têm demonstrado que a ocorrência das espécies ao longo do rio varia, até mesmo em regiões geograficamente próximas. No manguezal do estuário do rio Itabapoana, ES-RJ ( $\sim 60 \mathrm{~km}$ do estuário do rio Benevente), L. racemosa exibe maior abundância nos sítios com maior influência marinha em relação à Avicennia germinans, que por sua vez, exibe maior densidade relativa em áreas mais distantes do oceano (Bernini \& Rezende 2010). No manguezal do rio Paraíba do Sul ( 95km do estuário do rio Benevente), não se observaram tendências de distribuição ao longo do rio, provavelmente, em virtude do grande aporte de água doce (Bernini 2008). Bunt (1996) analisou 17 estuários na Austrália e constatou considerável variabilidade na distribuição das espécies ao longo dos rios. Esta variabilidade é atribuída às diferentes respostas de cada espécie aos fatores ambientais que operam em escalas que variam amplamente no tempo e no espaço. No entanto, tensores antrópicos também podem influenciar a distribuição das espécies ao longo do rio. Especificamente, em qualquer situação em que a floresta seja destruída localmente (ex. desmatamento, ciclone) e onde a superfície exposta possa ser adequada para qualquer uma das espécies, a flora da área regenerada pode ser dependente do que aconteceu com os diásporos das espécies (Bunt \& Stieglitz 1999), como por exemplo, a ordem de chegada junto aos sedimentos.

No estuário do rio Benevente, observa-se a presença de bancos arenosos com ausência de vegetação, principalmente próximo à embocadura do rio durante a maré baixa. Ao longo do estuário também são encontrados bancos de areia com presença de propágulos de todas as espécies, que em alguns casos se estabelecem e, posteriormente, constituem florestas mais jovens de mangue. A floresta analisada no sítio 10 (ilha), provavelmente se estabeleceu em função da deposição de areia no meio do rio. O assoreamento é resultante da má utilização do solo na agricultura e pecuária, com a retirada da mata ciliar ao longo da bacia do rio Benevente, podendo, futuramente, alterar a distribuição das espécies ao longo do rio.
A distribuição de troncos por classes de diâmetro exibiu formato do tipo jota invertido para a maioria das florestas e permitiu, juntamente com observações em campo, constatar a ausência de corte seletivo na área analisada. A distribuição do tipo jota invertido também foi reportada para outros manguezais (Jiménez \& Sauter 1991, Souza \& Sampaio 2001, Soares et al. 2003, Bernini \& Rezende 2004, Bernini \& Rezende, 2010). Este tipo de distribuição é registrado para comunidades que apresentam mortalidade natural relacionada ao amadurecimento da floresta, sendo indicativo de floresta saudável (Soares et al. 2003). As florestas que não exibiram este tipo de distribuição apresentaram um padrão mais homogêneo de distribuição dos troncos. De acordo com Smith (1992), florestas maduras tendem a exibir este tipo de comportamento, como observado nos sítios $\mathrm{S} 1$, S5, S6, S7, S8, S11 e S20 do presente estudo.

A espécie dominante registrada no estuário do rio Benevente, a A. schaueriana, é a mesma documentada por Espinoza \& Rosa (2009) para o manguezal do Rio Tavares (SC). No entanto, a espécie dominante varia de manguezal para manguezal em virtude das características abióticas e bióticas (ex. tolerância à salinidade e à inundação, competição interespecífica), além da influência de tensores naturais e antrópicos. Por exemplo, Matni \& Menezes (2006), descrevendo a estrutura de três florestas de mangue sob pouca influência antrópica na península de Bragança (PA), constaram a dominância de $R$. mangle. Em florestas de mangue do estuário de rio Paraíba do Sul (RJ), submetidas à baixa salinidade de água intersticial, Bernini \& Rezende (2004) verificaram que A. germinans foi a espécie dominante. No manguezal da Lagoa da Tijuca (RJ), local altamente impactado por várias atividades humanas, L. racemosa apresentou os maiores valores de dominância em locais alterados em processo de recomposição (Soares 1999).

A maior contribuição em área basal viva na classe diamétrica $\geq 10 \mathrm{~cm}$ na maioria dos sítios (exceto o $\mathrm{S} 15$ ) é característico de florestas de mangue maduras com elevado desenvolvimento estrutural. Os resultados encontrados estão de acordo com o estudo desenvolvido por Pereira et al. (2009) na embocadura do rio Benevente, onde se constatou que, com exceção de uma parcela que apresentou contribuição de $1 \%$ na classe entre 5,0 e $10,0 \mathrm{~cm}$ de diâmetro, a contribuição em área basal na classe diamétrica $\geq 10,0 \mathrm{~cm}$ foi de $100 \%$ nas parcelas analisadas, indicando o elevado desenvolvimento estrutural das florestas. Os valores registrados para os parâmetros estruturais encontram-se dentro da faixa observada para outros manguezais brasileiros (Soares 1999, Soares et al. 2003, 2008, Bernini \& Rezende 2004, 2010, Abreu et al. 2006). Entretanto, quando comparado a manguezais do Estado do Espírito Santo, a floresta do estuário do rio Benevente exibiu valores mais elevados de altura, DAP médio e área basal em relação ao manguezal do Fundão (Carmo et al. 1998b) e maiores valores de DAP médio e área basal em relação ao manguezal da Baía de Vitória (Carmo et al. 1995). Esta diferença pode ser atribuída às melhores condições ambientais (disponibilidade de água doce e nutrientes) e à menor influência antrópica (Lugo \& Snedaker 1974, Schaeffer-Novelli et al. 1990) no manguezal do rio Benevente em relação aos manguezais analisados Espírito Santo.

\section{Conclusão}

No manguezal do estuário do rio Benevente A. schaueriana e $R$. mangle exibiram dominância e maior densidade relativa em locais com maior influência marinha e maior período de inundação, respectivamente, enquanto que $L$. racemosa e A. germinans exibiram maior densidade relativa e dominância em locais com maior influência de água doce. A floresta analisada não exibe alterações estruturais causadas por corte seletivo e apresenta elevado desenvolvimento 
estrutural, uma vez que houve maior contribuição em área basal na classe maior que $10,0 \mathrm{~cm}$.

\section{Agradecimentos}

Ao Laboratório de Ciências Ambientais (LCA) do Centro de Biociências e Biotecnologia (CBB) da Universidade Estadual do Norte Fluminense Darcy Ribeiro (UENF) pela infra-estrutura necessária para o desenvolvimento deste estudo. Ao INCT - Instituto Nacional de Ciência e Tecnologia de Transferência de Materiais Continente-Oceano (Proc. 573.601/2008-9), pelo apoio financeiro. CER recebe apoio do CNPq (PQ 306.234/2007-6) e FAPERJ (E26/102.697/2008). À Prefeitura Municipal de Anchieta, ES. À Colônia de Pescadores Z-4 Marcílio Dias, Anchieta, ES.

\section{Referências Bibliográficas}

ABREU, M.O., MEHLIG, U., NASCIMENTO, R.E.S.A. \& MENEZES, M.P.M. 2006. Análise de composição florística e estrutura de um fragmento de bosque de terra firme e de um manguezal vizinhos na península de Ajuruteua, Bragança, Pará. Bol. Mus. Para. Emilio Goeldi 2(3):27-34.

BALL, M.C. 1980. Patterns of secundary sucession in a mangrove forest of southern Florida. Oecologia 44:226-235. http://dx.doi.org/10.1007/ BF00572684

BERNINI, E. 2008. Estrutura da cobertura vegetal e produção de serapilheira da floresta de mangue do estuário do rio Paraíba do Sul, Rio de Janeiro, Brasil. Tese de doutorado, Universidade Estadual do Norte Fluminense, Campos dos Goytacazes.

BERNINI, E. \& REZENDE, C.E. 2004. Estrutura da vegetação em florestas de mangue do estuário do rio Paraíba do Sul, Estado do Rio de Janeiro, Brasil. Acta Bot. Brasil. 18(3):491-502.

BERNINI, E. \& REZENDE, C.E. 2010. Variação estrutural em florestas de mangue do estuário do rio Itabapoana, ES-RJ. Biotemas 23(1):49-60.

BUNT, J.S. 1996. Mangrove zonation: an examination of data from seventeen riverine estuaries in tropical Australia. Ann. Bot. 78:333-341. http:// dx.doi.org/10.1006/anbo.1996.0128

BUNT, J.S. \& STIEGLITZ, T. 1999. Indicators of mangrove zonality: the Normanby river, N.E. Australia. Mangr. Salt Marsh. 3:177-184. http:// dx.doi.org/10.1023/A:1009951726930

CARMO, T.M.S., BRITO-ABAURRE, M.G., SENNA-MELO, R.M., ZANOTTI-XAVIER, S., COSTA, M.B. \& HORTA, M.M.M. 1995. Os manguezais da Baia Norte de Vitória, Espírito Santo: um ecossistema ameaçado. Rev. Brasil. Biol. 55(4):801-808.

CARMO, T.M.S., ALMEIDA, R., OLIVERIA, A.R. \& XAVIER, S.Z. 1998a Caracterização de um trecho do manguezal do rio da Passagem, Baía de Vitória, Vitória, ES, Brasil. In IV Simpósio de Ecossistemas Brasileiros. ACIESP, São Paulo, p.6-16.

CARMO, T.M.S., GÓES, P., ALMEIDA, A.P.L.S., SAMPAIO, F.D.F. \& ASSIS, A.M. 1998b. Caracterização do manguezal do rio Reis Magos, Fundão, Espírito Santo. In IV Simpósio de Ecossistemas Brasileiros. ACIESP, São Paulo, p.17-29.

CINTRÓN, G., LUGO, A.E., POOL, D.J. \& MORRIS, G. 1978. Mangrove of arid environments in Puerto Rico and adjacent islands. Biotropica 10(2):110-121. http://dx.doi.org/10.2307/2388013

CINTRON-MOLERO, G. \& SCHAEFFER-NOVELLI, Y. 1992. Ecology and management New World mangroves. In Coastal Plant Communities of Latin America (U. Seeliger, ed.). Academic Press, San Diego. p.233-258.

DUKE, N.C. 1992. Mangrove floristicts and biogeography. In Tropical mangrove ecosystems. Coastal and estuarine series (A.I. Robertson \& D.M. Alongi, eds). American Geoghysical Union, Washington. p.63-100.
ELLISON, A.M., MUKHERJEE, B.B \& ANSARUL, K. 2000. Testing patterns of zonation in mangroves: scale dependence and environmental correlates in the Sundarbans of Bangladesh J. Ecol. 88:813-824. http:// dx.doi.org/10.1046/j.1365-2745.2000.00500.x

ESPINOZA, H.C.F. \& ROSA, M.M.P.T. 2009. Evolução temporal da cobertura vegetal do manguezal do rio Tavares (Florianópolis-SC) empregando técnicas de sensoriamento remoto. In XIV Simpósio Brasileiro de Sensoriamento Remoto. INPE, Natal, p.2705-2712.

JIMÉNEZ, J.A. \& SAUTER, K. 1991. Structure and dynamics of mangrove forests along a flooding gradient. Estuaries 14(1):49-56. http://dx.doi. org/10.2307/1351981

LOVELOCK, C.E. \& FELLER, I.C. 2003. Photosynthetic performance and resource utilization of two mangrove species coexisting in a hypersaline scrub forest. Oecologia 134:455-462.

LUGO, A.E. \& SNEDAKER, S.C. 1974. The ecology of mangroves. Ann. Rev. Ecol. Syst. 5:39-64. http://dx.doi.org/10.1146/annurev. es.05.110174.000351

MAcNAE, W. 1968. A general account of the flora and fauna of mangrove swamps in the Indo-Pacific region. Adv. Mar. Biol. 6:73-270. http:// dx.doi.org/10.1016/S0065-2881(08)60438-1

MATNI, A. S. \& MENEZES, M.P.M. 2006. Estrutura dos bosques de mangue da Península de Bragança, Pará. Bol. Mus. Para. Emilio Goeldi 2(3):45-54.

McKEE, K.L. 1993. Soil physicochemical patterns and mangrove species distribution - reciprocal effects? J. Ecol. 81:477-487. http://dx.doi. org/10.2307/2261526

PEREIRA, F.V., FOLETTO, F., MOREIRA, T.M., GOMES, J.M.L. \& BERNINI, E. 2009. Estrutura da vegetação em duas áreas com diferentes históricos de antropização no manguezal de Anchieta, ES. Bol. Lab. Hidrobiol. 23(1):49-60.

SANTOS, M.C.F.V. 1986. Considerações sobre a ocorrência de Rhizophora harrisonii Leechamn e Rhizophora racemosa G.F.W. Meyer, no litoral do Estado do Maranhão, Brasil. Bol. Lab. Hidrobiol. 7:71-91.

SCHAEFFER-NOVELLI, Y. \& CINTRÓN, G. 1986. Guia para estudo de áreas de manguezal: estrutura, função e flora. Caribbean Ecologica Research, São Paulo.

SCHAEFFER-NOVELLI, Y., CINTRON-MOLERO, G. \& ADAIME, R.R. 1990. Variability of mangrove ecosystems along the brazilian coast. Estuaries 13(2):201-218. http://dx.doi.org/10.2307/1351590

SILVA, M.A.B., BERNINI, E. \& CARMO, T.M.S. 2005. Características estruturais de bosques de mangue do estuário do rio São Mateus, ES, Brasil. Acta Bot. Brasil. 19(3):465-471. http://dx.doi.org/10.1590/S010233062005000300006

SMITH, T.J. 1987. Effects of light and intertidal position on seedling survival and growth in tropical tidal forests. J. Exp. Mar. Biol. Ecol. 110:133-146. http://dx.doi.org/10.1016/0022-0981(87)90024-4

SMITH, T.J. 1992. Forest structure. In Tropical mangrove ecosystems. Coastal and estuarine series (A.I. Robertson \& D.M. Alongi, eds.). American Geophysical Union, Washington, p.101-136.

SNEDAKER, S.C. 1982. Mangrove species zonation: Why? In Tasks for vegetation science (D.N. Sen \& Rajpurohit, eds.). Dr. W. Junk Publishers, The Hague, p.111-125.

SOARES, M.L.G. 1999. Estrutura vegetal e grau de perturbação dos manguezais da Lagoa da Tijuca, Rio de Janeiro, RJ, Brasil. Rev. Brasil. Biol. 59(3):503-515.

SOARES, M.L.G., CHAVES, F.O., CORRÊA, F.M. \& SILVA JUINIOR, C.M.G. 2003. Diversidade estrutural de bosques de mangue e sua relação com distúrbios de origem antrópica: o caso da Baía de Guanabara (Rio de Janeiro). An. Inst. Geoc. 26:101-106.

SOARES, M.L.G., CHAVES, F.O., ESTRADA, G.C.D., CAVALCANTE, V.F., PORTUGAL, A.M.M. \& BARBOSA, B. 2008. Caracterização das florestas de mangue do complexo estuarino de Caravelas (Bahia, Brasil). Bol. Téc. Cient. 16(5):23-41. 
Petri, D.J.C. et al.

SOUZA, M.M. A \& SAMPAIO, E.V.S.B. 2001. Variação temporal da estrutura dos bosques de mangue de Suape - PE após a construção do porto. Acta Bot. Brasil. 15(1):1-12.

TOMLINSON, P.B. 1986. The botany of mangroves. Cambridge University Press, New York.

VALE, C.C. 1999. Contribuição aos estudos dos manguezais como indicadores biológicos das alterações geomórficas do estuário de rio São Mateus (ES). Dissertação de mestrado, Universidade de São Paulo, São Paulo.
VALE, C.C. \& FERREIRA, R.D. 1998. Os manguezais do litoral do Estado do Espírito Santo. In Simpósio de Ecossistemas da Costa Brasileira. ACIESP, São Paulo, p.88-94.

WATSON, J.G. 1928. Mangrove forests of the Malay Peninsula. Fraser \& Neave, Singapore.

WILLIAMS, W.T., BUNT, J.S. \& CLAY, H.J. 1991. Yet another method of species-sequencing. Mar. Ecol. Progr. Ser. 72:283-287. http://dx.doi. org/10.3354/meps072283

Recebido em 04/11/2010

Versão reformulada recebida em 15/02/2011

Publicado em 13/05/2011 